\title{
Research on a New Model and Network Coding Algorithm for Orthogonal Frequency Division Multiplexing System
}

\author{
Yong Luo ${ }^{1,2, *}$ \\ ${ }^{1}$ School of Software Engineering, Chongqing University, Chongqing 400044, China \\ ${ }^{2}$ Chongqing City Management College, Chongqing 401331, China
}

\begin{abstract}
The paper studies a new model and network coding algorithm for orthogonal frequency division multiplexing (OFDM) system. Cooperation diversity consists on using relay nodes to combat the effects of fading channels by means of spatial diversity. Physical-layer network coding is used to make the two-way s relay network achieving higher bandwidth efficiency than the traditional four-phase and three-phase bidirectional relaying schemes. Analogy Network Coding (ANC) has been already applied to OFDM for broadband wireless communications. In order to achieve high order of spatial diversity to combat fading, multi-antenna relay can be deployed. However, as the multiple antennas are geographically co-located, the received signals on each individual antenna may experience dependent fading. An alternative spatial diversity approach is through multi-relay cooperation, which poses other design challenges, e.g., synchronization, if all the relays participate in the transmission. As a result, Relay Selection has been proposed to achieve full diversity through a multi-relay network at a lower implementation cost.
\end{abstract}

Keywords: Dynamic interaction, Multi-relay network, Network coding algorithm, Orthogonal frequency division multiplexing system.

\section{INTRODUCTION}

Nowadays data networks are everywhere. If some years ago mobile phones took part in what could be called a communication revolution, this is the time for the mobile and wireless Internet technology to lead the role in another and more efficient way of connecting people. As the number of users grows up and their demands become more exigent, bandwidth gets scarcer.

New schemes have been developed while they aimed to providing a better use of the always limited bandwidth in the wireless networks. Network Coding constitutes one of those approaches. Network Coding provides a scheme to use bandwidth in a more efficient way for multi-cast networks. Initially, it was proposed to be employed in the networklayer for combining and processing packets before they used to be forwarded. Recently, a new approach has been proposed, which consists in applying network coding to the Physical Layer of a communication network called Physicallayer Network Coding (PNC). It has been shown that by applying PNC, bandwidth efficiency can be duplicated.

Moreover, this study also works on Cooperation Diversity (CD) schemes that consist in strategies aimed to mitigate the effects of fading channels by means of relaying nodes within the network.

*Address correspondence to this author at the School of Software Engineering, Chongqing University, Chongqing 400044, China;

Tel: +86-13996206329; E-mail: 241209@qq.com
One of these schemes named Opportunistic Relaying has drawn considerable attention recently because of its potential to improve the performance of wireless networks via proper relay selection algorithms designed to achieve better error performance.

Another challenge to be faced comes from the issue that modelling channel frequency response when broadband services are demanded, requires to take into account fading, multi-path, even shadowing. Finally, the multi-carrier scheme to be used will be Orthogonal Frequency Division Multiplexing (OFDM) because of two reasons: its popularity among wireless data networks and the issue that channel estimation is of vital importance for OFDM/PSK (Phase Shift Keying) modulation scheme when using coherent detection to achieve better performance. This work compares opportunistic relaying for single carrier and OFDM systems; we propose a relay selection algorithm and evaluate its performance through its asymptomatic Symbol Error Rate (SER) analysis.

\section{THE RELATED WORK}

Cooperation diversity schemes make use of relaying nodes to mitigate the effects of fading channels through spatial diversity $[1,2]$. By implementing PNC that was firstly proposed in [3], the relay network can achieve higher bandwidth efficiency than the traditional four-phase and threephase bi-directional relaying schemes. To ease implementation, simple PNC schemes such as Analogy Network Coding 
(ANC) with Amplify and Forward (AF) protocol were presented in [4]. In [5], ANC was applied to OFDM for broadband wireless communications.

Spatial diversity was presented as an effective way to deal with the multipath fading problem since geographically independent signals experience independent fading [1]. Multiple relay cooperation was proposed as an alternative to achieve spatial diversity without having physical arrays of antennas. However, multiple relay networks with all relays Participating (AP) in the communication pose other design challenges, e.g., synchronization. As a result, relay selection algorithms are more attractive in practice to make these networks achieve full diversity at a lower implementation cost.

In order to achieve high order of spatial diversity to combat fading, multiple antennas can be added to the relay node. However, as the multiple antennas are geographically colocated, the received signals on each individual antenna will experience dependent fading [1]. An alternative spatial diversity approach is through multiple relay cooperation, which poses other design challenges, e.g., synchronization, if we require all relays to participate in the communication. As a result, relay selection has been proposed to achieve full diversity through a multi-relay network at a lower implementation cost [6]. Relay selection algorithms for one way AF single carrier networks were recently studied in [6].

Max-Min was used as a selection criterion. Asymptotic SER analysis showed that it can achieve a diversity order of $\mathrm{R}+1$, where $\mathrm{R}$ is the number of relays and the additional diversity of order one was due to the direct path between source and destination. General SER expressions for one way AF single carrier networks were derived in [7], and a relay selection scheme was proposed based on maximization of the harmonic mean of the fading coefficients' average power. In [8], multiple relay selection schemes were proposed.

Selection of more than one relay was also discussed. The selection criteria considered include: maximum Signal to Noise Ratio (SNR) path [9, 10], relay-source distance, best harmonic mean [7], etc. However, no asymptotic SER analysis was given for more insightful design guidance. Several relay selection schemes for two-way AF networks were summarized in [11], and an asymptotic SER approximation was developed for a max-min suboptimal selection criterion. In [11], the optimal and sub-optimal relay selection criteria for two-way AF networks were proposed, and both criteria have been shown to achieve full diversity order. While most previous works focus on single carrier system, two relay selection approaches for one way OFDM network were proposed in [12], namely carrier-based and OFDM symbolbased selection criteria. Performance analysis in terms of the Pairwise Error Probability (PEP) showed both schemes can achieve full diversity order [13]. In [14], relay pre-coding and OFDM subcarrier grouping were proposed, but only simulation results were presented without performance analysis. In [15] cyclic prefix handling schemes are provided.

To the best of our knowledge, there is limited work in literature on relay selection for two-way AF OFDM networks. This motivates us to study this problem to propose practically viable relay selection algorithms and evaluate their performance through asymptotic SER analysis. We propose to maximize the smallest destination SNR as the core of our strategy to provide selection diversity. We define the destination SNR as the end-to-end SNR for each of the users, as the channel gains source-relay, relay-source are different. Unlike previous works, we base our criterion on a formal hypothesis supported by a mathematical principle which states that asymptotic behaviour of decreasing polynomial functions is mainly determined by the minimum exponent. Therefore, our hypothesis establishes that high SNR SER performance is mainly determined by the worst destination SNR conditions. Analytical and simulation results verified our approach.

\section{ORTHOGONAL FREQUENCY DIVISION MULTI- PLEXING AND COOPERATIVE DIVERSITY}

OFDM [5] is a technique to get a better use of the bandwidth in order to transmit a certain amount of information. Instead of the traditional approach in which one carrier is used to bear all the information, in the OFDM approach, many carriers are used to distribute the information among them. As many carriers are used, generally, more bandwidth is required; nevertheless, at the end it comes to be an improvement in the bit data rate since information is less vulnerable as it travels through different carriers.

In other words, dispersive broadband channels are converted into parallel narrow band sub-channels. OFDM is used to reduce the frequency-selective effects that multi-path channels have on wireless communications. OFDM consists in having a set of orthogonal carriers each of which will carry different information. Both transmitter and receiver are able to put the information in the right carrier and to take it from where it should be taken. As carriers are orthogonal to each other, sub-channels do not interfere each other.

OFDM is widely used nowadays in wireless data communications systems, as well as $4 \mathrm{G}$ cellular technology, DVB-T, etc. OFDM is easy to implement, requiring only two steps: Inverse Discrete Fourier Transform (IDFT) and Cyclic Prefix (CP) addition. IDFT can be implemented using the socalled Fast Fourier Transform (FFT) available with many software packages. The $\mathrm{CP}$ is what makes OFDM robust towards multi-path fading. The cyclic structure allows the linear convolution between discrete signals look like a circular convolution and then, thanks to the theorem that states that a convolution in the time domain is a multiplication in the frequency domain, signals can be multiplied pair-wise in the frequency domain without interfering each other. Fig. (1) illustrates this situation.

In Fig. (1), in the upper plot a circular convolution of two discrete signals is depicted. In the lower plot, a linear convolution between a signal with a cyclic structure and another discrete signal is depicted. As it can be seen, both resulting signals are equivalent provided that the latter gets sampled properly.

In order to sample the resulting signal properly, one has to remove the cyclic prefix and then trim the signal accordingly to remove the last elements that are not part of the corresponding circular convolution. When doing two-way relay 
communications, the cyclic structure of all the signals have to be kept if multipath channels are to be considered from sources to relay and backwards. In order to keep the cyclic structure of AF signal, some schemes have been proposed in [15]; here in this work we will use the second scheme.

The two cyclic prefix schemes explained in [15] are as follows: the first scheme consists in doubling the size of the cyclic prefix at the sources and removing half of it at the relay. Therefore, a cyclic prefix of twice the number of taps of the multi-path fading channel is used. The second cyclic prefix scheme (and the one we will use here) consists in using a cyclic prefix that is equal to the number of taps of the multipath fading channel and then removing it at the relay once it has added up the two signals coming for the sources. After amplifying the resulting signal, the relay then adds a new cyclic prefix which length is equal to the number of taps of the multi-path channel. The cyclic prefix is finally removed at the sources when the information is recovered.

Cooperative diversity consists in having single-antenna terminals around the receiving node such that they can form a virtual or distributed multi antenna system overcoming the packing problem of MIMO systems. Fading has been traditionally tackled using MIMO systems, although antenna separation must be guaranteed in order to avoid interference. Cooperative diversity allows reducing fading since information is sent over different nodes/paths/channels according to the relay terminals available.

Figs. (2 and 3) illustrate the idea of cooperative diversity. In the first figure we have two-way relay networks with multiple relay terminals. In the second figure we have plotted the Bit Error Rate (BER) performance against the SNR. As we can see the more relays participate in the communication, the better BER performance can be achieved.

It has been shown that cooperative diversity networks achieve diversity order equal to the possible number of paths between source and destination. Diversity order is the relationship between the SER and the SNR in the high SNR regime and it corresponds to the slope of the SER vs. SNR curve in the semi-logarithmic scale. That is, diversity order of $\mathrm{N}$ means that SER is proportional to the $n$th power of (1/SNR). In order to evaluate the performance of CD networks, Symbol Error Probability (or SER or BER has to be computed for different SNR scenarios. In [11], authors derive general average Symbol Error Probability expressions for one way AF relaying networks at high SNR regime. They develop an important analytical methodology to derive high SNR approximations that will be useful later on to derive similar expression for two-way networks.

\section{SYSTEM MODEL}

Fig. (4) illustrates the system model for any carrier where $\mathrm{j} \in\{0,1, \ldots \ldots, \mathrm{N}-1\}$. Relays perform AF protocol, information exchange is carried out within two time slots, channels are modelled as complex Gaussian (Rayleigh channels), for each carrier, flat fading is assumed and fading coefficients are considered constant within the time each frame lasts and independent from each frame to another. Channel gains are independent of each other, perfect CSI is assumed and gains are reciprocal, i.e., gain from source to relay is the same as the gain backwards.

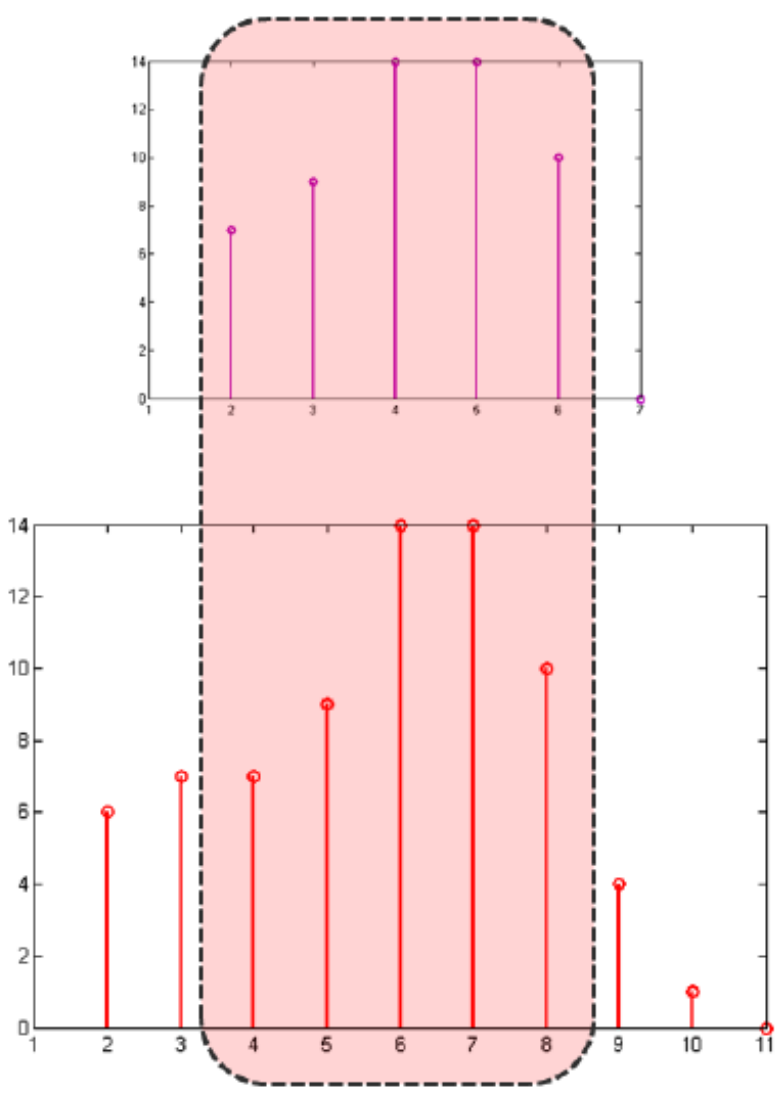

Fig. (1). Circular Convolution of two discrete signals and linear Convolution of a $\mathrm{CP}$ signal and the channel signal, before $\mathrm{CP}$ removal.
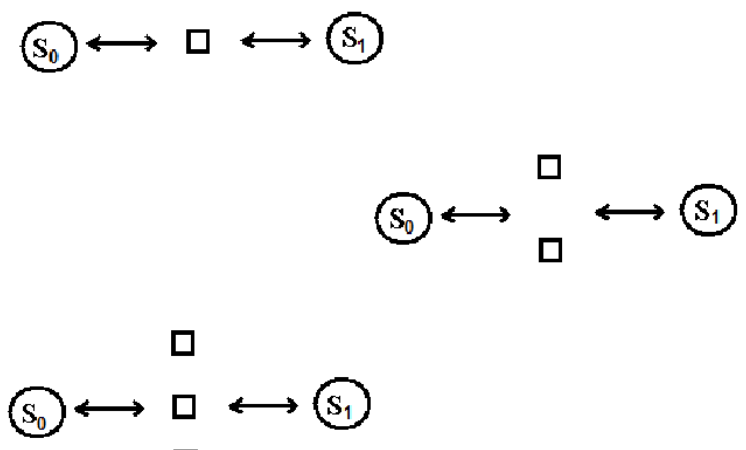

Fig. (2). Two-way relay networks with different number of relays cooperating in the communication.

OFDM modulation is accomplished in two steps, IDFT and $\mathrm{CP}$ addition, be $\mathrm{s}$ the BPSK-modulated signal form the source $i$. 


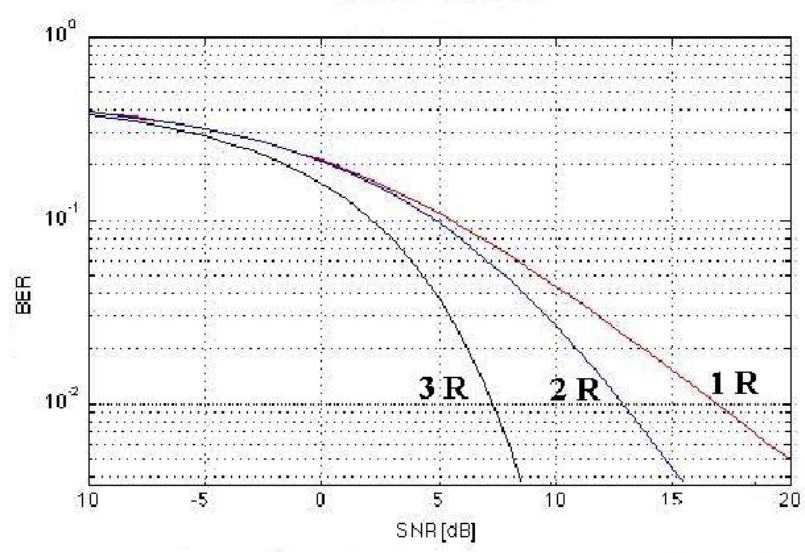

Fig. (3). BER performance against SNR for two-way relay networks with different number of relay nodes.

We considered a two-way relay network with $\mathrm{R}$ relays and two sources SO and S1. Each relay is equipped with a single antenna and implements the $\mathrm{CP}$ processing explained in (scheme II). Information exchange is accomplished within two time slots: in the first phase, both sources send simultaneously the information to all the relay nodes, each one receiving a superimposed signal; in the second phase one relay is chosen to forward an amplified version of the received signal back to the source nodes. Multipath $\mathrm{L}$ taps channels are considered and perfect CSI is assumed at both relay and sources. For a large number of taps, multipath channels are modeled as circular symmetric complex normal vectors with zero mean and identity covariance matrix.

We will do the signal analysis in a carrier basis as the OFDM signals are equipped with cyclic prefix making them resilient to multipath fading channels, at the time the linear convolution when the signals cross the channels are seen as pair-wise multiplications in the time domain provided that they are correctly sampled and leading to zero inter carrier interference.

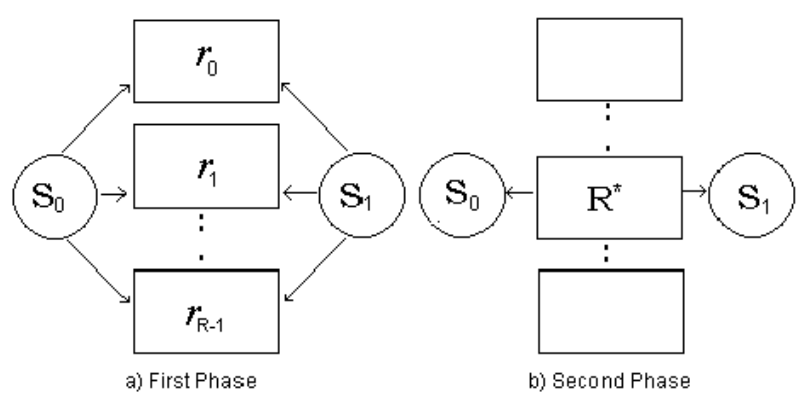

Fig. (4). System model single carrier.

Signal received by the relay ' $r$ ', at carrier ' $c$ ':

$y_{r, c}=\sqrt{\rho_{s}} H_{0} S_{0}+\sqrt{\rho_{s}} H_{1} S_{1}+n_{r, c}$

The signal received by the source 0 after cancelling the self-information is: $y_{0, r, c}=\sqrt{\rho_{s} \rho_{r}} \beta_{r, c} H_{0} H_{1} S_{1}+\sqrt{\rho_{r}} \beta_{r, c} H_{0} n_{r, c}+n_{0, r, c}$

From (2) we compute the SNR as follows:

$$
y_{0, r, c}=\frac{\left|\sqrt{\rho_{s} \rho_{r}} \beta_{r, c} H_{0} H_{1}\right|}{\operatorname{var}\left\{\sqrt{\rho_{r}} \beta_{r, c} H_{0} n_{r, c}+n_{0, r, c}\right\}}
$$

The Probability Density Function PDF of (3) is given by

$$
f_{\gamma, r, c}=\xi \cdot e^{-\xi x} U(x)
$$

Figs. (5 and 6) illustrate forward network. Two approaches the system model for the OFDM two-way amplify and are forward the entire block and, another depicted, one in which only one relay is selected to approach in which each carrier chooses their best relay, therefore multiple relays are selected and all of them participate forwarding different data.

OFDM is implemented within two steps: Inverse Discrete Fourier Transform IDFT and Cyclic Prefix addition. In order to keep the OFDM structure within the network, the relay node implements a Cyclic Prefix processing that consists on removing it once it superimpose the signals and adds another cyclic prefix to the new structure once the signal is amplified in order to forward it back to the sources.

This section provides a mathematical and graphical description of the system model. Channel is modelled here and OFDM scheme implementation is discussed as well as the Cyclic Prefix (CP) handling issue.

We consider a two-way relay network with $\mathrm{R}$ relays and two sources S0 and S1 as illustrated in Fig. (1). Information exchange is accomplished within two time slots. In the first slot, both sources send their information simultaneously to all the relay nodes, and each relay receives a superimposed signal. In the second slot one relay is chosen to forward an amplified version of its received signal to the two source nodes. Each relay is equipped with a single antenna and implements cyclic prefix (CP) processing scheme 2 as explained in [16], which guarantees a cyclic structure in the transmitted signal from the relay node in the second slot. We assume L-tap multipath channel for all the links and perfect knowledge of channel state information (CSI) at all the odes. As proven, when the number of taps matches the Fast Fourier Transform (FFT) size N, both frequency and time domain channels can be modelled as circular symmetric complex normal random vectors with zero mean and identity covariance matrix.

\section{SIMULATION RESULT}

Set up parameters: source/relay powers, modulation (BPSK, QPSK...), and number of carriers, number of taps, number of relays, and number of minimum errors to compute the BER.

Generate $2 \mathrm{R}$ independent, identically distributed, complex normal channel. Real and imaginary parts are normally distributed. 


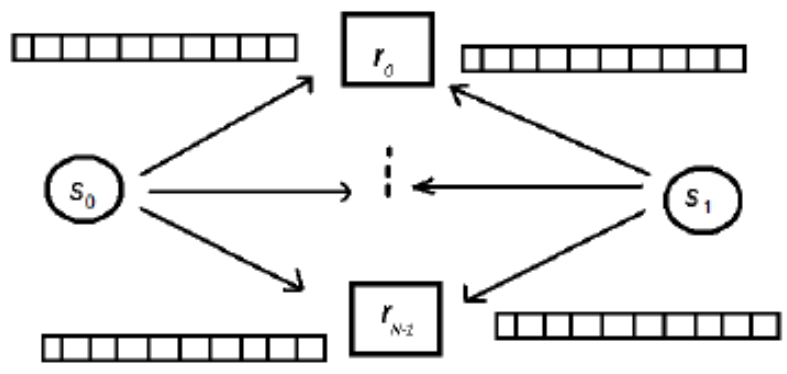

a) First Time Slot

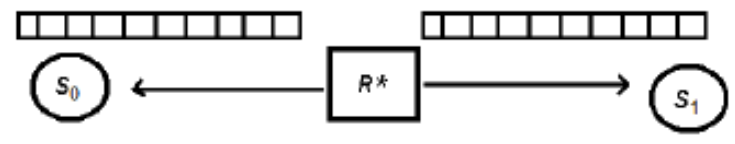

b) Second Time Slot

Fig. (5). OFDM Model block-based approach.

Channels are normalized to have unit variance.

Generate a SNR array and loop over different SNR values, for each value:

1) Generate source signals

2) Send signals to all relays

3) Choose the best relay according to the criterion

4) Do AF from the selected relay back to the sources

5) Do self-information removal at each source

6) Do equalization

7) Do hard decision (or any other decision process, such as Maximum Likelihood).

8) Compare original data with received data and compute the number of errors

9) If the number of errors is greater than or equal to the minimum number or errors enough to compute the
BER, then

(a) Compute BER for this SNR and repeat from step 1 for the NEXT SNR value.

If not:

Stay on the CURRENT SNR value and accumulate the number of errors until the minimum is reached.

Once BER is computed for each SNR value, then, results are plotted. Analytical results are computed from the theoretical expression and they are also plotted for comparison.

We present a simulation of an OFDM relay network to verify the proposed selection algorithm and asymptotic SER performance. Source and relay powers were set to 1 and BPSK modulation was implemented, channel was modelled as a 4-taps channel, and cyclic prefix length and FFT size were set to 4. Fig. (7) compares all three relay selection algorithms presented in this paper: Max-Min, Max-Min-Min and optimal. As expected, carrier-based Max-Min selection outperforms block-based Max-Min-Min selection except for the single-relay case, for which, both approaches lead to the same results.

It is clear from the Fig. (7) that Max-Min-Min criterion has almost the same SER performance as the optimal blockbased relay selection criterion. Fig. (7) also shows that at high SNR, the closed form asymptotic analytical SER match the simulation results; therefore, it verifies the analytical treatment. As the number of relay increases, the SER performance also improves, due to higher diversity gain.

\section{CONCLUSION}

Relay selection criteria for two-way AF OFDM networks were presented as well as their performance analysis. Simulation results verified the proposed asymptotic approximation. Carrier-based approach was also analysed and simulated; results were similar to single carrier systems performance. Carrier-based selection showed to perform better than block-based, according to the comparison carried out. MaxMin- Min (block-based) selection criterion performed close to the optimal single relay selection approach, so did the

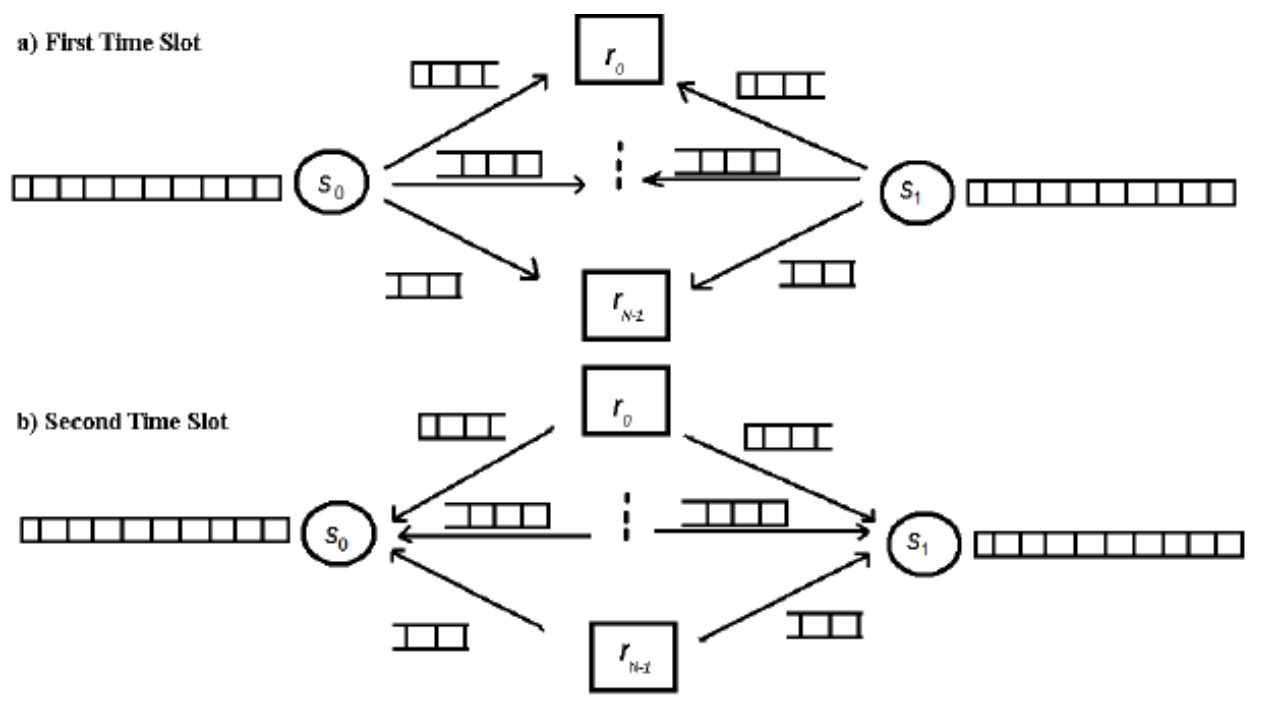

Fig. (6). OFDM System Model carrier-based approach. 


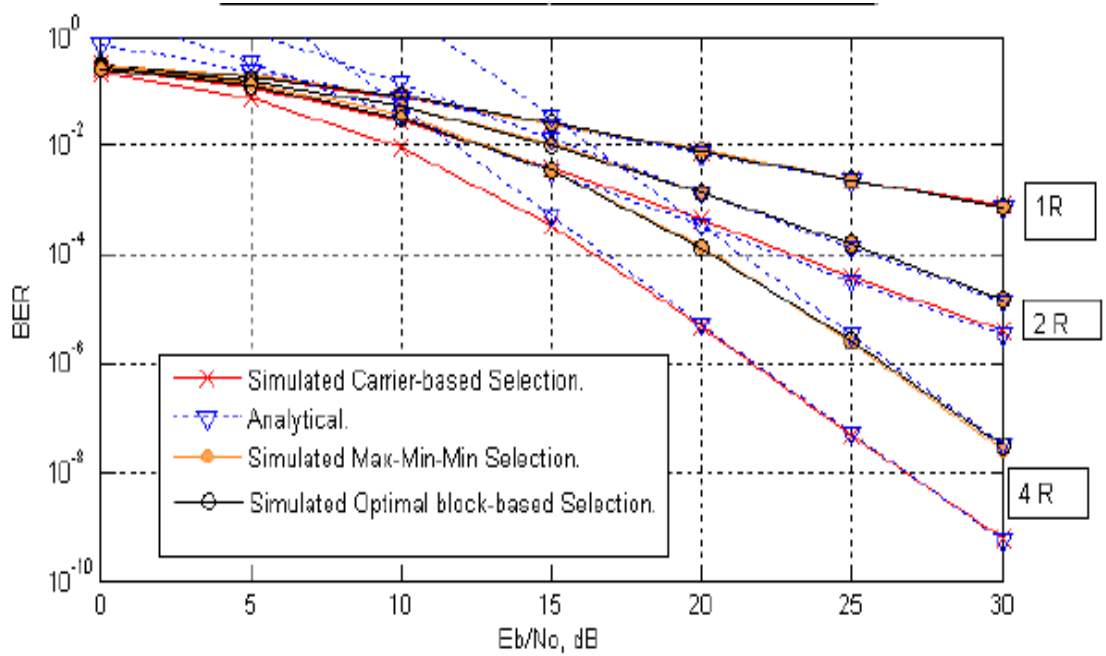

Fig. (7). Carrier-based vs. Block-based Selection in the high SNR.

Max-Min (carrier-based) compared to the single-carrier optimal relay selection criterion. The higher diversity order is the result of the improvement of the statistical properties of the weakest destination SNR of the network, given that the SER performance depends mainly on the worst conditions and the high-SNR asymptotic behaviour is indeed dependent on the lowest diversity order.

The theoretical treatment can be applicable to cases in which the channel is modelled with less taps than the number of carriers, according to the simulations carried out.

\section{CONFLICT OF INTEREST}

The author confirms that this article content has no conflicts of interest.

\section{ACKNOWLEDGEMENTS}

This work is supported by the Natural Science Foundation Project of CQ CSTC: 2011BB2064.

\section{REFERENCES}

A. Sendonaris, E. Erkip and B. Aazhang, "User cooperation diversity. Part I. System description" IEEE Transactions on Communications, vol. 51, no. 11, pp. 1927-1938, 2003

[2] A. Sendonaris, E. Erkip and B. Aazhang, "User cooperation diversity. Part II. Implementation Aspects and performance analysis", IEEE Transactions on Communications, vol. 51, no. 11, pp. 19391948, 2003.

[3] S. Zhang, S.C. Liew and P.P. Lam, "Hot Topic: Physical-Layer Network Coding", Proceedings of the $12^{\text {th }}$ Annual International Conference on Mobile Computing and Networking, MobiCom, pp. 358-365, 2006.

[4] S. Katti, S. Gollakota and D. Katabi, "Embracing wireless interference: analog network coding", SIGCOMM Computer Communication Reviews, vol. 37, pp. 397-408, 2007.
[5] H. Gacanin and F. Adachi, "Broadband analog network coding", IEEE Transactions on Wireless Communications, vol. 9, no. 5, pp. 1577-1583, 2010.

[6] P. Yeoh, M. Elkashlan, Z. Chen and I. Copings, "SER of multiple amplify-and-forward relays with selection diversity", IEEE Transactions on Wireless Communications, vol. 59, no. 8, pp. 2078 2083, 2011.

[7] A. Ribeiro, X. Cai and G Giannakis, "Symbol error probabilities for general Cooperative links", IEEE Transactions on Wireless Communications, vol. 4, no. 3, pp. 1264-1273, 2005.

[8] Y Jing and H. Jafarkhani, "Single and multiple relay selection schemes and their diversity orders", IEEE Transactions on Wireless Communications, vol. 8, no. 3, pp. 1414-1423, 2009.

[9] E. Koyuncu, Y Jing, and H. Jafarkhani, Distributed beamforming in wireless relay networks with quantized feedback, IEEE Journal on Selected Areas in Communications, vol. 26, no. 8, pp. 1429-1439, 2008.

[10] Y. Zhao, R. Adve and T.J. Lim, "Symbol error rate of selection amplify and- forward relay systems", IEEE Communications Letters, vol. 10, no. 11, pp. $757-759,2006$

[11] L. Song, "Relay selection for two-way relaying with amplify and forward protocols", IEEE Transactions on Vehicular Technology, vol. 60 , no. 4, pp. 1954-1959, 2011

[12] B. Gui, L. Dai and L. Cimini, Selective relaying in cooperative OFDM systems: Two-hop random network, Wireless Communications and Networking Conference, pp. 996-1001, 2008.

[13] Y Ding and M. Uysal, "Amplify-and-forward cooperative OFDM with multiple-relays: performance analysis and relay selection methods", IEEE Transactions on Wireless Communications, vol. 10 no. 8, pp. $4963-4968,2009$.

[14] Q. Zhang, F. Shu, M. Wang and J. Sun, "Relay selection schemes for precoded cooperative OFDM and their achievable diversity orders", IEEE Signal Processing Letters, vol. 18, no. 4, pp. 231-234, 2011

[15] S. Sun, C. Yuen and T. Q. Quek, "An analog space-time block coded OFDM wireless relay systems", $12^{\text {th }}$ International symposium on wireless personal multimedia communications, 2009

[16] M. Hasna and M.-S. Alouini, "End-to-end performance of transmission systems with relays over Rayleigh-fading channels", IEEE Transactions on Wireless Communications, vol. 2, no. 6, pp. 11261131, 2003. 